Supporting Information

\title{
Composite Quantum Sensors Based on Fluorescent Nanodiamonds for Intracellular Controlled Heating in Living Cells
}

Shingo Sotoma ${ }^{* \dagger, \dagger}$ and Yoshie Harada ${ }^{\dagger}, \|$

†Institute for Protein Research, Osaka University, Osaka 565-0871, Japan

$\ddagger$ Japan Society for the Promotion of Science (JSPS), Tokyo 102-0083, Japan

॥Quantum Information and Quantum Biology Division, Institute for Open and Transdisciplinary Research Initiatives, Osaka University, Osaka 565-0871, Japan

Corresponding Author: ssotoma@ protein.osaka-u.ac.jp 


\section{MATERIALS and METHODS}

Chemicals and Materials: Fluorescent nanodiamonds were purchased from FND Biotech. Hydrogen tetrachloroaurate-(III) tetrahydrate $\left(\mathrm{HAuCl}_{4} \cdot 4 \mathrm{H}_{2} \mathrm{O}, 99 \%\right)$ was purchased from FUJIFILM Wako Chemicals. Dopamine hydrochloride and glycidol were purchased from SigmaAldrich. Glycidyltrimethylammonium chloride (ca. $80 \%$ in water) was purchased from TCI Chemicals. Dulbecco's modified eagle medium (DMEM), 0.25\% Trypsin-EDTA, PBS, penicillin and streptomycin, and fetal bovine serum were purchased from Thermo Fisher Scientific.

Synthesis of FND-PDA: FND-PDA was prepared based on the previously reported method but with a slight modification. ${ }^{1}$ FNDs $(100 \mu \mathrm{g})$ was suspended in $1 \mathrm{ml}$ of a dopamine hydrochloride solution with different concentrations $(20,50,100,200$, and $500 \mu \mathrm{g} / \mathrm{ml})$ buffered to $\mathrm{pH} 8.5$ using $10 \mathrm{mM}$ Tris-HCl. The suspension was shaken for $24 \mathrm{~h}$, and then washed via centrifugation with Milli-Q water twice at $12 \mathrm{krpm}$ for $5 \mathrm{~min}$, yielding FND-PDA.

Synthesis of FND-PDA-GNP: FND-PDA $100 \mu \mathrm{g}$ were dispersed in different concentrations of the $\mathrm{HAuCl}_{4}$ aqueous solution $(5,10,50,100,200$, and $500 \mu \mathrm{g} / \mathrm{ml})$ followed by sonication for 3 min. The mixture was heated to $90^{\circ} \mathrm{C}$ for 20 min, yielding FND-PDA-GNP.

Synthesis of FND-PDA2-GNP: FND-PDA-GNP were prepared $1 \mathrm{mg}$ by scaling up the above protocol, and then suspended in $10 \mathrm{ml}$ of a dopamine hydrochloride solution $(50 \mu \mathrm{g} / \mathrm{ml})$ buffered to $\mathrm{pH} 8.5$ using $10 \mathrm{mM}$ Tris- $\mathrm{HCl}$. The suspension was shaken for $24 \mathrm{~h}$, and then washed via centrifugation with Milli-Q water twice at $12 \mathrm{krpm}$ for 5 min, yielding FND-PDA2-GNP.

Synthesis of FND-PDA2-GNP-HPG: FND-PDA2-GNP (1 mg) was dispersed in glycidol (2 ml), sonicated, and stirred for $20 \mathrm{~h}$ at $90^{\circ} \mathrm{C}$ under a nitrogen atmosphere. The resulting gel was diluted 
using Milli-Q water and then centrifuged at $12 \mathrm{krpm}$ for $10 \mathrm{~min}$ to collect the FND-PDA2-GNPHPG. FND-HPG was then centrifuged and washed three times to remove the free glycidol and HPG.

Synthesis of FND-PDA2-GNP-HPGTMA: FND-PDA2-GNP (1 mg) was dispersed in a mixture solution of glycidol and glycidyltrimethylammonium chloride at a volume ratio of 1.5/0.5 (ml/ml). Note that the water in GTMA was removed by rotary evaporation before use. The mixtures were sonicated and then stirred for $20 \mathrm{~h}$ at $90^{\circ} \mathrm{C}$ under a nitrogen atmosphere. After the reaction, the resulting gel was diluted with Milli-Q water and then centrifuged at $12 \mathrm{krpm}$ for $10 \mathrm{~min}$ to collect the FND-PDA2-GNP-HPGTMA. Then they were washed three times to remove the by-products and unreacted materials.

Characterization: TEM images were obtained using an H-7650 (Hitachi) operated at an $80 \mathrm{kV}$ accelerating voltage. UV-visible (vis) spectra were recorded on a V-560 spectrometer (JASCO) operating in the region of $400-850 \mathrm{~nm}$ with a resolution of $1 \mathrm{~nm}$. Zeta potentials of the samples in neutral water were measured using a Zetasizer Nano ZSP (Malvern). Fourier transform infrared (FT-IR) spectra of the dried samples were recorded on an FT/IR 6100 (JASCO) with a resolution of $1 \mathrm{~cm}^{-1}$ based on attenuated total reflectance method.

Cell culture and observation under a confocal fluorescence microscope: HeLa cells were cultured in 35 mm glass-based dished with Dulbecco's modified Eagle's medium supplemented with $10 \%$ fetal calf serum and $1 \%$ antibiotic-antimycotic at $37{ }^{\circ} \mathrm{C}$ with $5 \% \mathrm{CO}_{2}$. The CQS samples were dispersed in culture medium with various concentrations $(10,20,50$, and $100 \mu \mathrm{g} / \mathrm{ml})$ and then incubated for $30 \mathrm{~min}$. After extensive wash by PBS, the cells were observed under a fluorescence microscope (Leica SP8). 


\section{Temperature measurements using FNDs through optically detected magnetic resonance}

microscopy: The temperatures were measured using FNDs and a home-built microscope, which was reported previously. ${ }^{2}$ Briefly, a continuous neodymium-doped yttrium aluminum garnet (Nd:YAG) laser at $532 \mathrm{~nm}$ illuminated the FNDs in FND-PDA-GNP or the cCQS to initialize and read out the spin state of the NVCs on an inverted microscope system (Nikon, Ti-E). The fluorescence from the NVCs was captured using an oil-immersion objective lens (Nikon SR HP Apo TIRF, 100×, NA 1.49), passed through a long-pass filter to cut off the excitation light, and was imaged using an electron-multiplying charge-coupled device camera (EMCCD, Andor, iXon860). A two-turn copper coil with a diameter of approximately $1 \mathrm{~mm}$ was placed just above the coverslip to irradiate the sample with microwaves (MWs) at frequencies near the electron-spin resonance in the NVCs. A gate pulse provided by a pulser (Berkley Nucleonics Corporation, 565) activated a MW generator (Agilent, E8257D) to output a MW pulse, which was then amplified by linear MW power amplifiers (Mini-Circuits, PAN35-5A and ZHL-16W-43+) and then transmitted through a coaxial cable to a MW coil. The pulser and the image acquisition by the EMCCD camera were synchronized using a computer (Dell T3500, XT, USA) with LabVIEW software (National Instruments, TX, USA). ODMR spectra were recorded for a range of MW frequencies as the difference between the fluorescence intensities with and without MW irradiation. The temperature of the sample was set at $300 \mathrm{~K}$ using a stage-top incubator (TOKAI HIT, TPi-108RH26) on an electric moving stage.

Evaluation of the photothermal effect of FND-PDA-GNP: Fluorescence images were recorded as the MW frequency was digitally swept across the resonant frequency range from 2855 to 2885 $\mathrm{MHz}$ in increments of $0.3 \mathrm{MHz}$. Through this process, ODMR spectra were obtained from the single FND-PDA-GNP or cCQS particles. The exposure time of the camera was $5 \mathrm{~ms}$. The number 
of 64 images ( 2 images for cell experiment) were accumulated per MW frequency, and two scans (32 scans for cell experiment) were performed. Then, each ODMR spectrum was fitted to the sum of the two Lorentzian functions to determine the zero-filed splitting, $D$. We note that $D$ is defined as the central value of the two resonances for $m_{s}=0$ to 1 and $m_{s}=0$ to -1 . The measured temperature change was determined based on the previously reported thermal shift in the ODMR peak, $\Delta D / \Delta T=-0.074 \mathrm{MHz} /{ }^{\circ} \mathrm{C}$, and we estimated the temperature rise of the FND encapsulated in PDA-GNP as a function of the laser intensity. A 532-nm laser was used for both the ODMR measurement and GNP heating. The temperature rise at the laser intensities was estimated in reference to the excitation at $7 \mathrm{~mW}$.

Spearman's rank correlation coefficient: The Spearman's rank correlation coefficient (SRCC) is used to discover the strength of a link between two sets of data. SRCC is given by

$$
r_{s}=1-\frac{6 \sum_{i=1}^{n} d_{i}^{2}}{n\left(n^{2}-1\right)}
$$

where $d_{i}$ is the difference in ranks for $\mathrm{x}_{\mathrm{i}}$ and $\mathrm{y}_{\mathrm{i}}$, and $n$ is the number of observations. The interpretation of $r_{s}$ is displayed in Table S1. In the manuscript, an SRCC analysis was applied to evaluate the data for Figure S4 and Figure S5.

Monte Carlo error estimation: To estimate the temperature accuracy from an ODMR signal, we performed the following Monte Carlo error simulation. First, we measured the ODMR spectrum and then fitted the spectrum using two Lorentzian functions (Figure S5C and S5D). The temperature was then determined from the resonant point in the ODMR spectrum (see the Experimental section). Next, we calculated the noise level in the ODMR spectrum in addition to its standard deviation $(\sigma)$. We hypothesized that the noise in the ODMR spectrum is always distributed as a Gaussian with a standard deviation of $\sigma$. Then we added a randomly drawn error 
to the fitted ODMR spectrum. After 500 random (Monte Carlo) simulations, we obtained the distribution of the resonant frequencies in the spectrum (Figure S5E and S5F), which indicated the temperature error. This distribution was fitted to a Gaussian and its standard deviation was determined. From the results, we estimated the temperature error for each spectrum. 


\section{Supporting Figures}
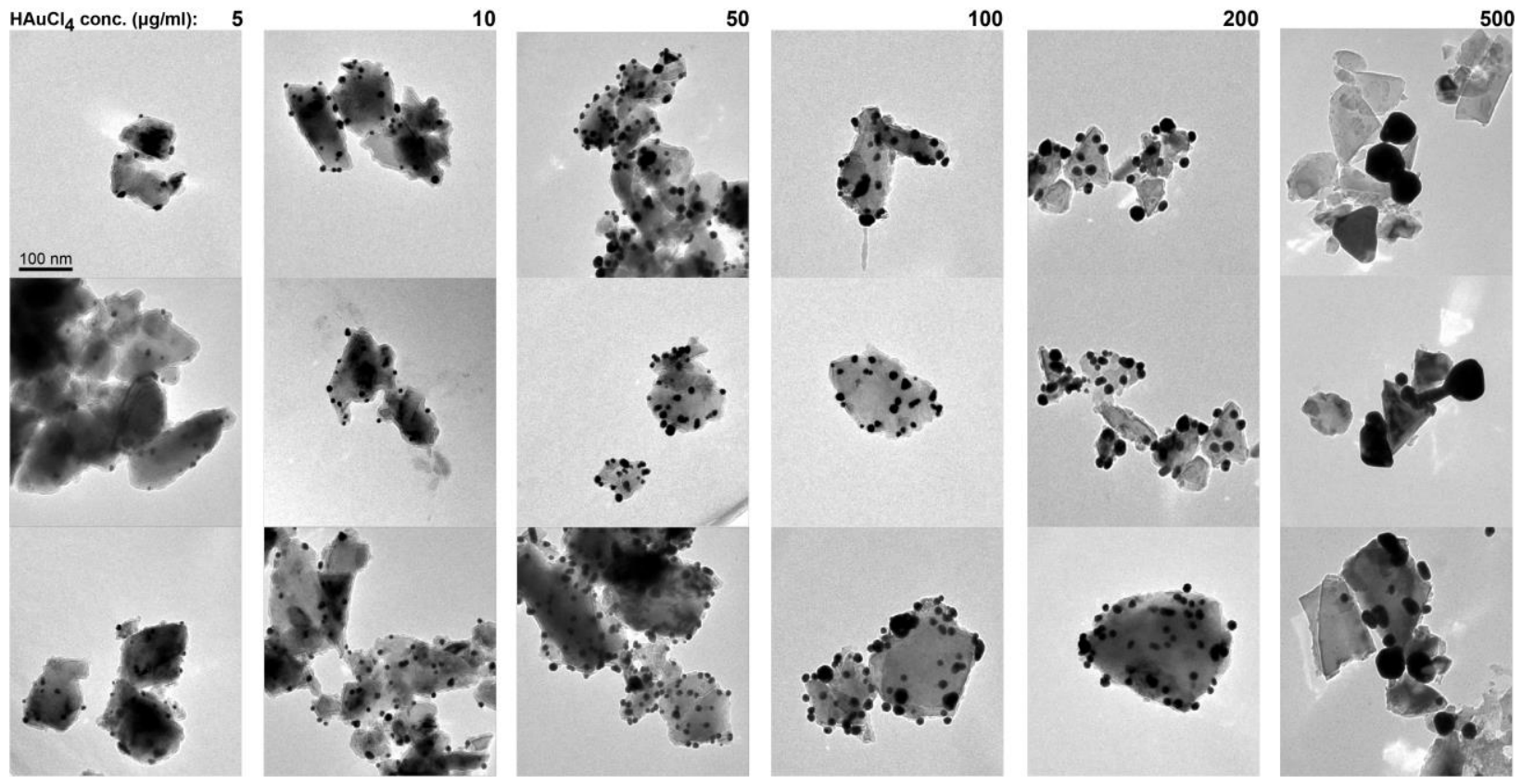

Figure S1. TEM images of the FND-PDA-GNP prepared with different concentrations of the $\mathrm{HAuCl}_{4}$ aqueous solution. The conditions are displayed on top of the images, and three images are shown for each condition. Note that the FND-PDA prepared with $50 \mu \mathrm{g} / \mathrm{ml}$ dopamine hydrochloride was used for the synthesis.

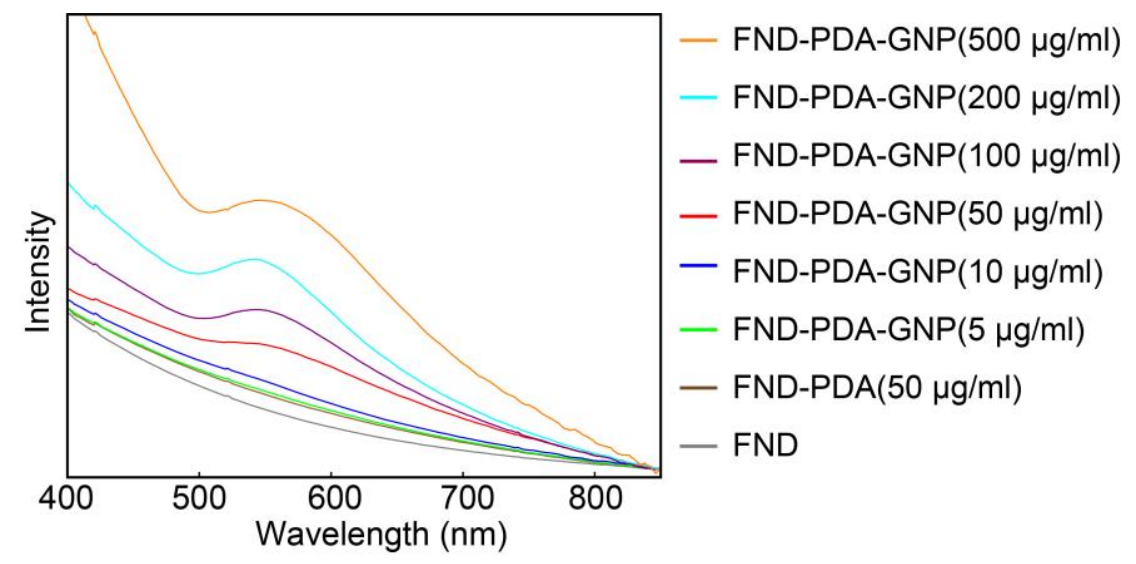

Figure S2. UV-vis absorption spectra of the samples. The concentrations are shown on the right side of the panel. 


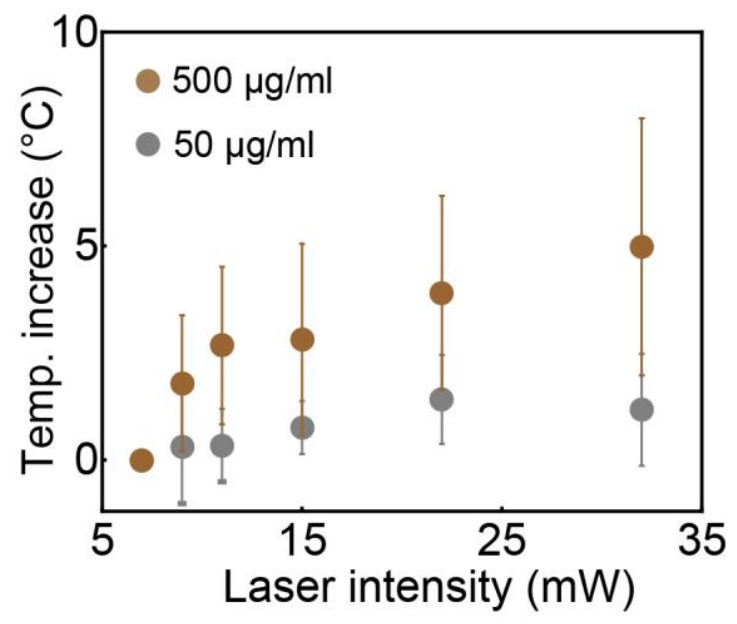

Figure S3. Correlation between the temperature increase and laser power of the FND-PDA prepared with 50 (grey) and 500 (brown) $\mu \mathrm{g} / \mathrm{ml}$ dopamine hydrochloride solution. The panel shows the corresponding mean values (circles) and sample standard deviations (bars).

A

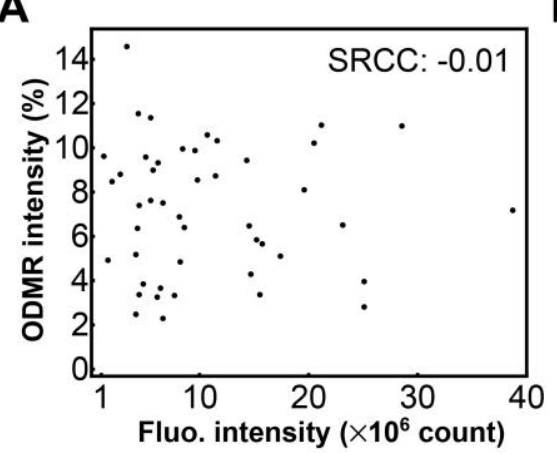

B

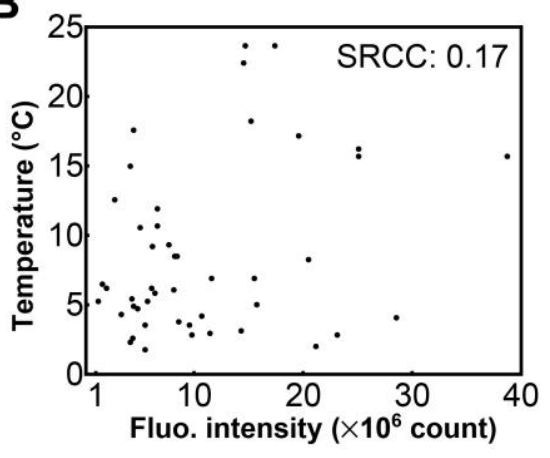

Figure S4. Single-particle plots for the (A) ODMR intensity or (B) temperature increase as a function of the fluorescence intensity when excited by $7 \mathrm{~mW}$. The SRCC values are shown at each panel. 

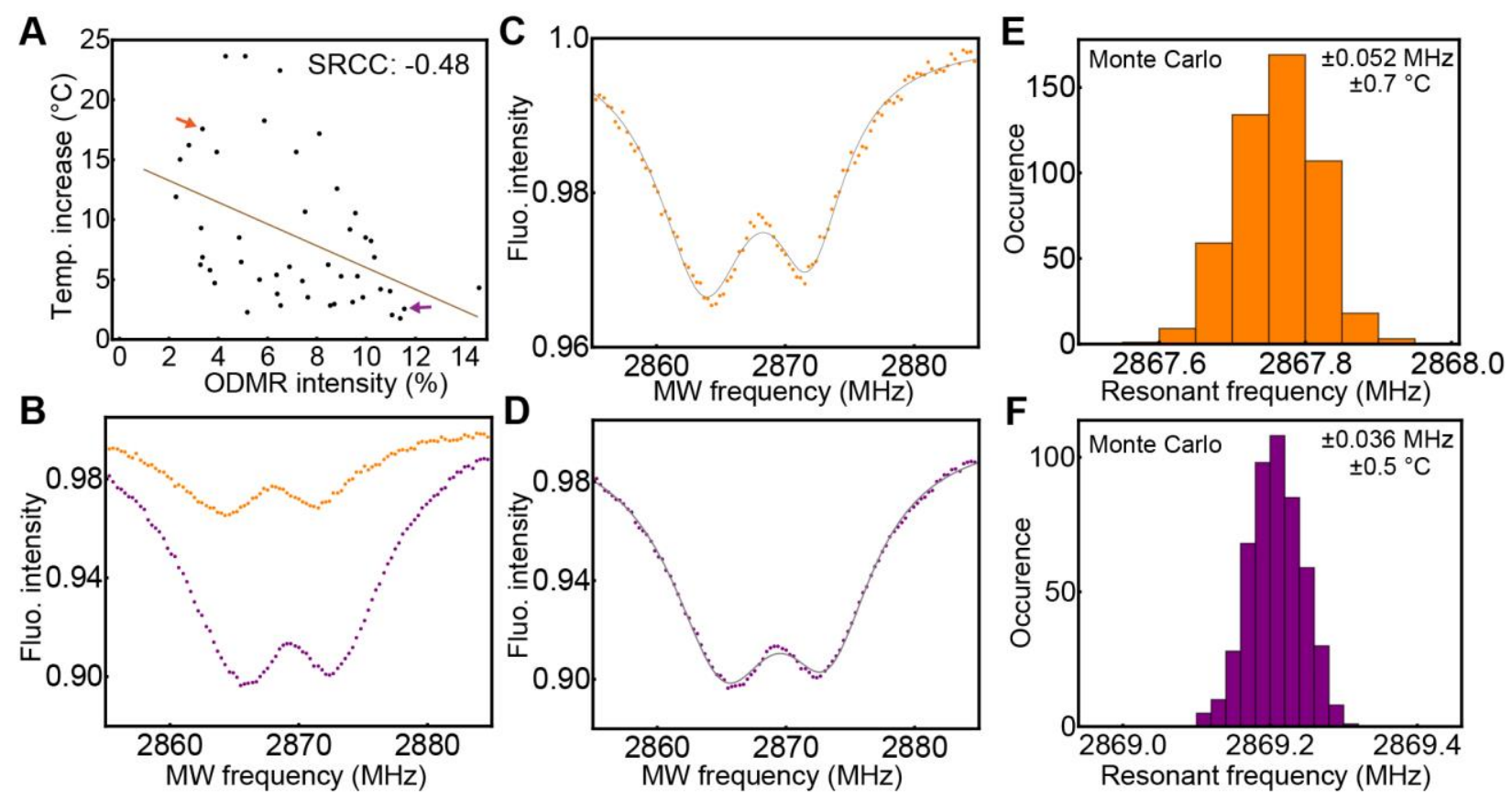

Figure S5. (A) Single-particle plots for the temperature increase as a function of the ODMR intensity when excited by $7 \mathrm{~mW}$. The SRCC value is shown at the panel. (B) The two ODMR spectra are indicated by arrows in (A). The same color of the arrows shown in (A) corresponds to the same colored ODMR spectra in (B). (C) and (D) are the ODMR spectrum in (B) accompanied with Lorentzian fittings. (E) and (F) are the Monte Carlo error estimations of (C) and (D), respectively. The errors of the $D$ value and temperature are shown in each panel.

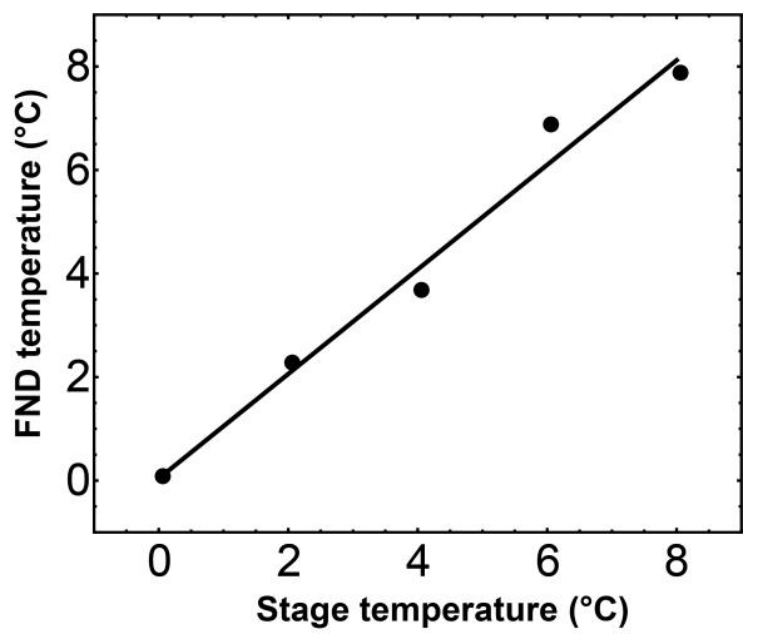

Figure S6. The measured temperature change of FND-PDA-GNP when the surrounding temperature is controlled using a stage heater. 


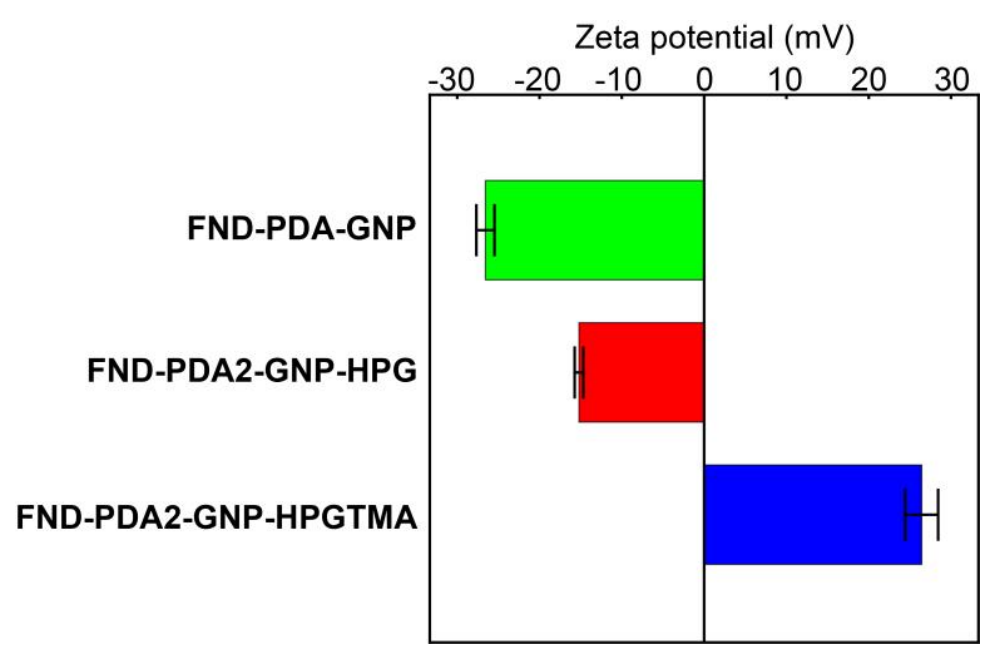

Figure S7. Zeta potential values of the samples.

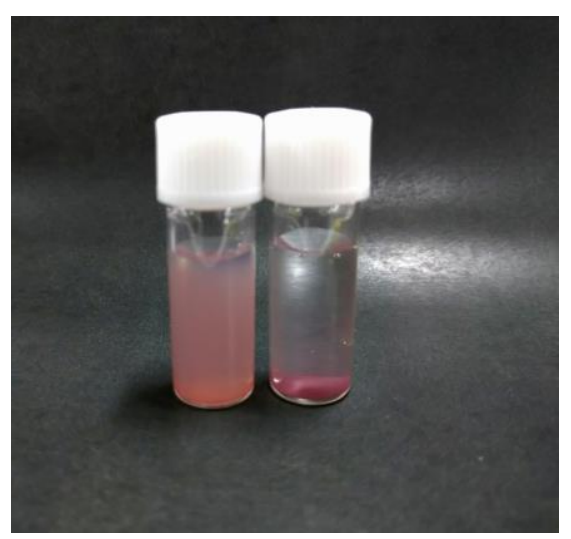

Figure S8. Picture of FND-PDA-GNP (right) and FND-PDA2-GNP-HPG (left) dispersed in $1 \mathrm{M}$ $\mathrm{NaCl}$ aqueous solution after $24 \mathrm{~h}$. 


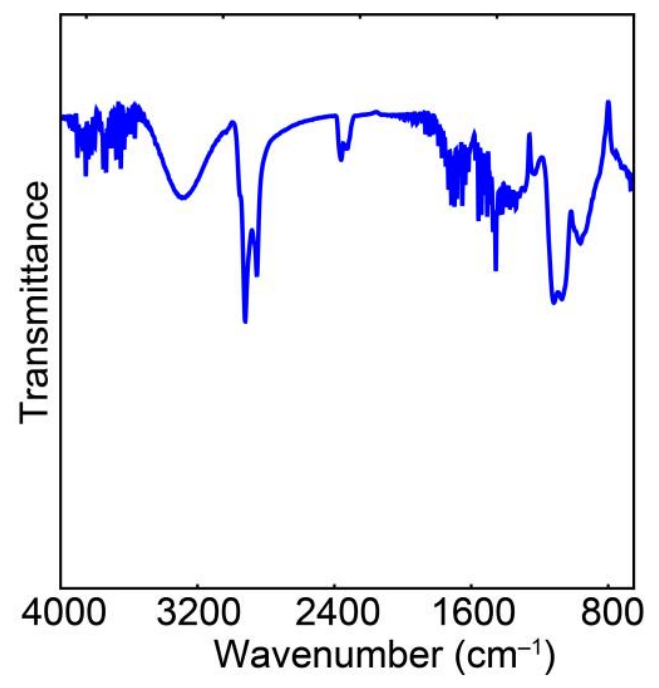

Figure S9. FTIR spectrum of FND-PDA2-GNP-HPGTMA. 
TABLE

Table S1. Interpretation of Spearman's rank correlation coefficients $\left(r_{s}\right)^{3,4}$

\begin{tabular}{cc}
\hline $\boldsymbol{r}_{\boldsymbol{s}}$ & Interpretation \\
\hline 0.70 to $1.00(-0.70$ to -1.00$)$ & Very strong positive (negative) correlation \\
0.40 to $0.69(-0.40$ to -0.69$)$ & Strong positive (negative) correlation \\
0.30 to $0.39(-0.30$ to -0.39$)$ & Moderate positive (negative) correlation \\
0.20 to $0.29(-0.20$ to -0.29$)$ & Weak positive (negative) correlation \\
0.01 to $0.19(0$ to -0.19$)$ & No or negligible correlation \\
\hline
\end{tabular}




\section{REFERENCES}

(1) Jung, H.-S.; Cho, K.-J.; Seol, Y.; Takagi, Y.; Dittmore, A.; Roche, P. A.; Neuman, K. C. Polydopamine Encapsulation of Fluorescent Nanodiamonds for Biomedical Applications. Advanced Functional Materials 2018, 28, 1801252.

(2) Sekiguchi, T.; Sotoma, S.; Harada, Y. Fluorescent Nanodiamonds as a Robust Temperature Sensor inside a Single Cell. Biophysics and Physicobiology 2018, 15, 229-234.

(3) Leclezio, L.; Jansen, A.; Whittemore, V. H.; de Vries, P. J. Pilot Validation of the Tuberous Sclerosis-Associated Neuropsychiatric Disorders (Tand) Checklist. Pediatr Neurol 2015, 52 (1), $16-24$.

(4) Dancey, D.; Reidy, J. Statistics without Maths for Psychology: Using SPSS for Windows. London, England: Prentice-Hall; 2004 